\title{
Non-mechanical scanning laser Doppler velocimetry with sensitivity to direction of transverse velocity component using optical serrodyne frequency shifting
}

\author{
Koichi Maru* and Kento Watanabe \\ Department of Electronics and Information Engineering, Kagawa University \\ 2217-20 Hayashi-cho, Takamatsu, Kagawa 761-0396, Japan \\ Tel: +81-87-864-2230, Fax: +81-87-864-2262, E-mail address: maru@eng.kagawa-u.ac.jp
}

\begin{abstract}
This paper proposes a non-mechanical axial scanning laser Doppler velocimeter (LDV) with sensitivity to the direction of the transverse velocity component using optical serrodyne frequency shifting. Serrodyne modulation via the electro-optic effect of a $\mathrm{LiNbO}_{3}(\mathrm{LN})$ phase shifter was employed to discriminate the direction of the transverse velocity component. The measurement position is scanned without any moving mechanism in the probe by changing the wavelength of the light input to the probe. The experimental results using a sensor probe setup indicate that both the scan of the measurement position and the introduction of directional sensitivity are successfully demonstrated.
\end{abstract}

\section{Keywords}

Laser Doppler velocimeter; laser sensors; optical sensors; interferometry 


\section{Introduction}

Velocity distribution measurement has been an essential technology in many researches and industries. A differential laser Doppler velocimeter (LDV) is a standard apparatus for measuring fluid flows or moving objects because of its noninvasive nature, small measurement volume giving fine spatial resolution, and a linear response. To measure velocity distribution, various techniques for mechanically scanning the measurement position for a differential LDV have been reported [1-6]. In these techniques, a moving mechanism is used in transmitting optics, e.g. a movable lens [1], moving mirrors [2-4], a galvanometer-type scanner [5], or a rotating transparent plate [6]. For axial scanning, a scanning range of 60-200 cm from the output lens was reported by using a movable lens [1], and $10 \mathrm{~mm}$ by using rotating mirrors [3]. For a practical use, an LDV with a compact sensor probe, which can be easily handled and is generally separated from the main body, is desirable. Several non-scanning LDVs with sensor probes have been reported [7-10]. To realize a scanning LDV with a compact and reliable sensor probe, we have proposed scanning LDVs without any moving mechanism in their probes [11-15]. In these non-mechanical scanning LDVs, the measurement position is scanned by changing the wavelength input to the probe, and diffraction gratings are used inside the probe instead of a moving mechanism. These LDVs have advantages because they are durable against mechanical impact on the probe and free from abrasion compared with conventional scanning techniques using a moving mechanism in the probe.

To apply the proposed scanning technique to most velocity measurement applications, introducing directional sensitivity, i.e. measurement of the sign of the velocity component, is indispensable. For introducing directional sensitivity into an LDV, optical frequency shifting $[8,13,16-22]$ is commonly used. The fiber-optic LDV with a compact probe capable of providing directional information has been proposed [8], although this LDV was a non-scanning type. We have proposed a non-mechanical scanning LDV with directional sensitivity [13]. In these LDVs, Bragg cells using the acousto-optic effect were employed for optical frequency shifting. However, typical Bragg cells are bulky and require high-power RF sources.

Optical serrodyne modulation is another promising method to obtain direct optical frequency shifting. Serrodyne modulation via the electro-optic effect of a $\mathrm{LiNbO}_{3}(\mathrm{LN})$ phase shifter does not require high-power signals. In addition, waveguide-based LN phase-shifter chips are commercially available, and a beam can be easily input and output using optical fibers via butt coupling between the chip and fiber. This advantage contributes to simplifying the structure for optical frequency shifting and realizing LDVs based on fiber optics compared with the use of Bragg cells. Several LDVs with directional sensitivity by optical serrodyne modulation have been reported [18,19,21,22], although these LDVs were for single-point velocity measurement.

In this paper, we propose a non-mechanical scanning LDV in which optical serrodyne frequency shifting using an $\mathrm{LN}$ phase shifter is employed to discriminate the direction of the 
transverse velocity component. The introduction of directional sensitivity and the scanning function are experimentally demonstrated using a sensor probe setup.

\section{Concept}

The concept of the proposed non-mechanical scanning LDV is illustrated in Fig. 1. This LDV consists of a main body including a tunable laser and LN phase shifter, and a probe including transmitting and receiving optics. In the same manner as the axial scanning LDVs reported in $[11,13]$, the measurement position is axially scanned by the wavelength change of the light input to the probe by using diffraction gratings in the transmitting optics. The beam from the tunable laser is split into two beams with a polarization beam splitter (PBS). One of the beams is directly input to the probe, and the other is input to the LN phase shifter to shift its optical frequency by serrodyne modulation. The two beams are input to the probe via two polarization maintaining fibers (PMFs). In the probe, each beam is passing through each of two sets of the transmitting optics arranged symmetrically in which the beam is collimated and diffracted on the grating. The two beams cross each other at the measurement position. The scattered beams from the moving object are monitored with a photodiode (PD) via the receiving optics. The measurement position can be axially scanned when the wavelength changes because the directions of the two beams diffracted on the gratings change with the wavelength. Because the tunable laser and phase shifter can be separated from the probe, the probe can be kept simple and reliable.

To discriminate the direction of the transverse velocity component perpendicular to the axial direction at the measurement position, the frequency of one of the beams split with the PBS is preshifted by $f_{0}$ using the $\mathrm{LN}$ phase shifter driven by a sawtooth voltage signal with linear ramps and a nominal duty cycle of $100 \%$. The phase of the light propagating the phase shifter linearly changes with the applied voltage. Serrodyne frequency shifting is achieved when the peak-to-peak voltage of the sawtooth signal applied to the phase shifter, $V_{\mathrm{pp}}$, corresponds to $2 n \pi$ phase shift, where $n$ is an integer. Then, the preshift frequency $f_{0}$ is given by $n f_{s}$, where $f_{s}$ is the frequency of the sawtooth signal. Then, the beat frequency monitored with the PD is biased by $f_{0}$, and the resulting beat frequency is the sum of $f_{0}$ and the Doppler shift at the measurement position, $f_{D}$, given by [23]

$$
f_{D}=2 v \sin \theta(\lambda) / \lambda
$$

where $v$ is the transverse velocity component, $\lambda$ is the wavelength, and $\theta(\lambda)$ is half the angle between the two beams at the measurement position. Because $v$ and $f_{D}$ have the same sign, the velocity component including its directional information can be derived from Eq. (1) using the measured $f_{D}$ as the difference between the monitored beat frequency and $f_{0}$.

\section{Experimental setup}


An experiment was performed to demonstrate the scan and directional sensitivity in the proposed LDV. The experimental setup is illustrated in Fig. 2. A tunable laser (ANDO AQ4321A) was used as a lightsource. The wavelength $\lambda$ was changed over $1525-1565 \mathrm{~nm}$. The beam from the laser was input to a polarization controller and PBS, and split into two beams. The splitting ratio of the two beams was roughly controlled with the polarization controller. One of the beams was directly launched into the transmitting optics in the sensor probe setup with the vertically polarized state. The other beam was input to the phase shifter consisting of a straight waveguide on a z-cut LN substrate before it was input to the probe setup. Here, the rotational angle of the input PMF was adjusted so that the input beam was coupled to the TM mode of the waveguide. The sawtooth signal was applied to the electrodes on the waveguide of the phase shifter with a signal generator (NF Corporation WF1948). To avoid dc drift, the bias voltage of the sawtooth signal was set to zero. The frequency-preshifted beam from the phase shifter was input to the probe setup also with the vertically polarized state. Each beam input to the sensor probe setup was passing through lenses and incident on one of reflection-type ruled diffraction gratings with a grating period of $1.67 \mu \mathrm{m}$. The incident angle on the gratings was set to $50^{\circ}$ where the angular dispersion of the first-order diffracted beam was $3.5 \times 10^{-2} \mathrm{deg} / \mathrm{nm}$ at $\lambda$ $=1545 \mathrm{~nm}$ [12]. Its first-order diffracted beam was incident on a target rotating in a vertical plane. The scattered beams on the surface of the target were detected with an InGaAs PD (Thorlabs PDA10CS). The beat signal was measured with a digital oscilloscope (Tektronix TDS2014C), and its spectrum was calculated with the fast Fourier transform (FFT). To determine the axial shift of the measurement position, the target was moved manually in the axial direction at the position where a peak of the beat signal appeared in its spectrum and its amplitude became a maximum. The vertical position of the target was set so that the distance between the measurement position and center of rotation was $18.3 \mathrm{~mm}$. The rotational speed of the target was monitored with an encoder and frequency counter. We set $\theta(\lambda)$ to $15^{\circ}$ and the distance between the grating and measurement position along the axial direction to $290 \mathrm{~mm}$ at $\lambda$ $=1545 \mathrm{~nm}$.

To investigate the condition of serrodyne modulation in this experiment, we measured the beat signal of the modulated and unmodulated lights using a setup of a Mach-Zehnder interferometer where the LN phase shifter was inserted in one arm. The spectra of the beat signal for $V_{\mathrm{pp}}=10.7 \mathrm{~V}$ and $\lambda=1545 \mathrm{~nm}$ are shown in Fig. 3. Here, the frequency of the sawtooth signal, $f_{s}$, was set to 0.5 and $1 \mathrm{MHz}$. Peaks corresponding to the fundamental harmonic (at $f_{s}$ ) and higher-order harmonics of the modulated optical signal were observed in each spectrum. The higher-order harmonics were minimized when $V_{\mathrm{pp}}=10.7 \mathrm{~V}$, which corresponds to $2 \pi$ phase shift. The amplitude ratio of the second-order harmonic (at $2 f_{s}$ ) to the fundamental harmonic at this voltage was less than $-29 \mathrm{~dB}$ for $f_{s}=0.5 \mathrm{MHz}$ and $-23 \mathrm{~dB}$ for $f_{s}=1 \mathrm{MHz}$ for the wavelengths of 1525,1545 , and $1565 \mathrm{~nm}$. In the experiment hereafter, we set $V_{\mathrm{pp}}$ to $10.7 \mathrm{~V}$ 
where $f_{s}$ was used as the preshift frequency $f_{0}$.

\section{Results and discussion}

Fig. 4 shows the measured spectra of beat signals for $f_{s}=1 \mathrm{MHz}$. Here, $\lambda=1525,1545$, and $1565 \mathrm{~nm}$, and the rotational speed of the target was $-6.0,0$, and $6.0 \mathrm{~s}^{-1}$. The peak with the maximum amplitude was located at the preshift frequency $f_{0}=f_{s}$ when the target was not rotated, and shifted with the rotation of the target. Similar behavior was observed also for $f_{s}=0.5 \mathrm{MHz}$. The Doppler frequency $f_{D}$ was estimated as the difference between the measured beat frequency (determined as the frequency where the maximum peak was located) and $f_{0}$. For each wavelength, the sign of $f_{D}$ coincided with the sign of rotational speed at the measurement position. This indicates that the sensitivity to the direction of the transverse velocity component was successfully obtained. A residual peak was observed at $f_{0}$ during the rotation of the target. This is probably attributed to the polarization coupling at the interfaces of the $\mathrm{LN}$ phase shifter and PMFs.

Fig. 5 shows the measured beat frequency as a function of wavelength for various rotational speeds of the target for $f_{s}=0.5$ and $1 \mathrm{MHz}$. Theoretical values derived from Eq. (1) are also shown in Fig. 5. The measured beat frequencies were in good agreement with the theoretical values for both preshift frequencies. At any wavelengths, the measured $f_{D}$ changed linearly with the change in the rotational speed, as appeared in standard laser Doppler velocimetry. The root mean square (rms) of the difference between the measured and theoretical values of $f_{D}$ was 1.1, $1.5,1.5$, and $2.5 \mathrm{kHz}$ for $f_{s}=0.5 \mathrm{MHz}$, and $3.0,1.5,1.7$, and $1.3 \mathrm{kHz}$ for $f_{s}=1 \mathrm{MHz}$, for the rotational speeds of $-6.0,-3.0,3.0$, and $6.0 \mathrm{~s}^{-1}$, respectively. These values correspond to the measurement error in velocity of $0.5-1.5 \%$.

The axial measurement position was measured by moving the target in the axial direction when the wavelength was changed. Fig. 6 plots the measured values of axial measurement positions as a function of wavelength. Here, $f_{s}$ was set to $0.5 \mathrm{MHz}$. The measurement was repeated six times. Theoretical values derived from a model based on an optical ray tracing $[11,13]$ are also plotted. The scanning function was successfully observed, i.e., the measurement position shifted with the wavelength change. The measured values agree well with the theoretical ones. The scanning range was estimated to be $28.3 \mathrm{~mm}$ over the wavelength range of $1525-1565 \mathrm{~nm}$. The standard deviation of the measured axial positions for each wavelength was $0.23-0.55 \mathrm{~mm}$. This deviation corresponds to the measurement error in the axial position of the maximum spectral peak. The theoretical value is within one standard deviation of the measured values for any wavelength.

The scanning range of $28.3 \mathrm{~mm}$ was about $9.8 \%$ of the working distance defined as the distance between the grating and measurement position along the axial direction. The scanning range is limited by some factors such as the wavelength range of the tunable laser and the 
characteristics of the gratings. The range can be further enhanced by increasing the wavelength range, reducing the crossing angle between the two beams at the measurement position, or using the condition of larger angular dispersion of the gratings. In this experiment, the incident angle on the gratings was set to $50^{\circ}$ because the angular dispersion is insensitive to the deviation in the incident angle. If the incident angle of $0^{\circ}$ is used, where the angular dispersion is $9.2 \times 10^{-2}$ $\mathrm{deg} / \mathrm{nm}$, the scanning range can be enhanced to 2.6 times of that in this experiment. This scanning range would be practical for velocity distribution measurement of flow in pipes as an example of applications.

The sensor probe setup in this paper consisted of bulk optical components. The probe should be miniaturized for practical applications as future works.

In this experiment the wavelength was not swept; the wavelength was set at intervals. The maximum wavelength sweep speed of the tunable laser used is $100 \mathrm{~nm} / \mathrm{s}$. For practical applications, a tunable laser that has a higher sweep speed would be desired. In the existing mechanical techniques, the scanning rates of several hundreds of $\mathrm{Hz}$ were reported in the mechanical scanning LDV using moving mirrors [4] or galvanometer-type scanner [5]. By using a fast wavelength-swept laser such as that reported in [24], the scanning rate comparable to or more than that in the mechanical techniques would be expected in the proposed method.

\section{Conclusion}

Optical serrodyne frequency shifting was employed to discriminate the direction of the transverse velocity component for the non-mechanical scanning LDV on the basis of wavelength change. The probe does not require any complicated optical system in it because the tunable laser and phase shifter can be separated from the probe. In contrast to frequency shifting using Bragg cells, serrodyne modulation via the electro-optic effect of an LN phase shifter does not require high-power RF source and is suitable for making fiber-optic LDVs. It was found that the measurement position shifted according to the wavelength change and the measured beat frequency corresponded to the velocity component including its directional information. The measurement error in velocity was estimated to be $0.5-1.5 \%$. The proposed scanning LDV would be useful for many applications in velocity measurement.

\section{Acknowledgments}

This work in part was supported by Adaptable and Seamless Technology Transfer Program through target-driven R\&D, Japan Science and Technology Agency. 


\section{References}

[1] G. R. Grant, K. L. Orloff, Appl. Opt. 12 (1973) 2913-2916.

[2] F. Durst, B. Lehmann, C. Tropea, Rev. Sci. Instrum. 52 (1981) 1676-1681.

[3] M. Uchiyama, K. Hakomori, J. Jpn. Soc. Precis. Eng. 48 (1982) 939-944 (in Japanese).

[4] P. Sriram, S. Hanagud, J. Craig, N. M. Komerath, Appl. Opt. 29 (1990) 2409-2417.

[5] K. A. Shinpaugh, R. L. Simpson, Meas. Sci. Technol. 6 (1995) 690-701.

[6] E. B. Li, A. K. Tieu, W. Y. D. Yuen, Opt. Lasers Eng. 35 (2001) 41-49.

[7] H. Nishihara, J. Koyama, N. Hoki, F. Kajiya, M. Hironaga, M. Kano, Appl. Opt. 21 (1982) 1785-1790.

[8] J. Knuhtsen, E. Olldag, P. Buchhave, J. Phys. E: Sci. Instrum. 15 (1982) 1188-1191.

[9] M. Stiegimeier, C. Tropea, Appl. Opt. 31 (1992) 4096-4105.

[10] H.-E. Albrecht, M. Borys, N. Damaschke, C. Tropea, Laser Doppler and Phase Doppler Measurement Techniques, Springer, Berlin, 2003, sec. 7.3.

[11] K. Maru, Opt. Express 19 (2011) 5960-5969.

[12] K. Maru, T. Fujiwara, R. Ikeuchi, Appl. Opt. 50 (2011) 6121-6127.

[13] K. Maru, T. Hata, Appl. Opt. 51 (2012) 4783-4787.

[14] K. Maru, IEEE Sens. J. 12 (2012) 2648-2652.

[15] K. Maru, T. Hata, Appl. Opt. 51 (2012) 8177-8183.

[16] T. Eiju, K. Matsuda, J. Ohtsubo, K. Honma, K. Shimizu, Appl. Opt. 20 (1981) 3833-3837.

[17] X. Shen, J. Zhang, Z. Wang, H. Yu, Acta Mechanica Sinica 2 (1986) 81-92.

[18] H. Toda, M. Haruna, H. Nishihara, J. Lightwave Technol. 5 (1987) 901-905.

[19] H. Toda, K. Kasazumi, M. Haruna, H. Nishihara, J. Lightwave Technol. 7 (1989) 364-367.

[20] H.-E. Albrecht, M. Borys, N. Damaschke, C. Tropea, Laser Doppler and Phase Doppler Measurement Techniques, Springer, Berlin, 2003, sec. 7.2.3.

[21] Y. Li, S. Meersman, R. Baets, Appl. Opt. 50 (2011) 2809-2814.

[22] Y. Li, S. Verstuyft, G. Yurtsever, S. Keyvaninia, G. Roelkens, D. Van Thourhout, R. Baets, Appl. Opt. 52 (2013) 2145-2152.

[23] H.-E. Albrecht, M. Borys, N. Damaschke, C. Tropea, Laser Doppler and Phase Doppler Measurement Techniques, Springer, Berlin, 2003, sec. 2.1.

[24] N. Fujiwara, R. Yoshimura, K. Kato, H. Ishii, F. Kano, Y. Kawaguchi, Y. Kondo, K. Ohbayashi, H. Oohashi, IEEE Photon. Technol. Lett. 20 (2008) 1015-1017. 


\section{Figure captions}

Fig. 1. Concept of proposed non-mechanical scanning LDV.

Fig. 2. Experimental setup.

Fig. 3. Measured spectra of beat signal of modulated and unmodulated lights using Mach-Zehnder interferometer setup. $V_{\mathrm{pp}}=10.7 \mathrm{~V}$ and $\lambda=1545 \mathrm{~nm}$. (a) $f_{s}=0.5 \mathrm{MHz}$. (b) $f_{s}=1$ MHz.

Fig. 4. Measured spectra of beat signals for $f_{s}=1$ MHz. (a) $\lambda=1525 \mathrm{~nm}$. (b) $\lambda=1545 \mathrm{~nm}$. (c) $\lambda$ $=1565 \mathrm{~nm}$. The rotational speed of the target was $-6.0,0$, and $6.0 \mathrm{~s}^{-1}$.

Fig. 5. Measured beat frequency as a function of wavelength for various rotational speeds. (a) $f_{s}$ $=0.5 \mathrm{MHz}$. (b) $f_{s}=1 \mathrm{MHz}$. Theoretical values are also plotted.

Fig. 6. Measured value of axial measurement position as a function of wavelength. $f_{s}=0.5 \mathrm{MHz}$. Theoretical values are also plotted. 


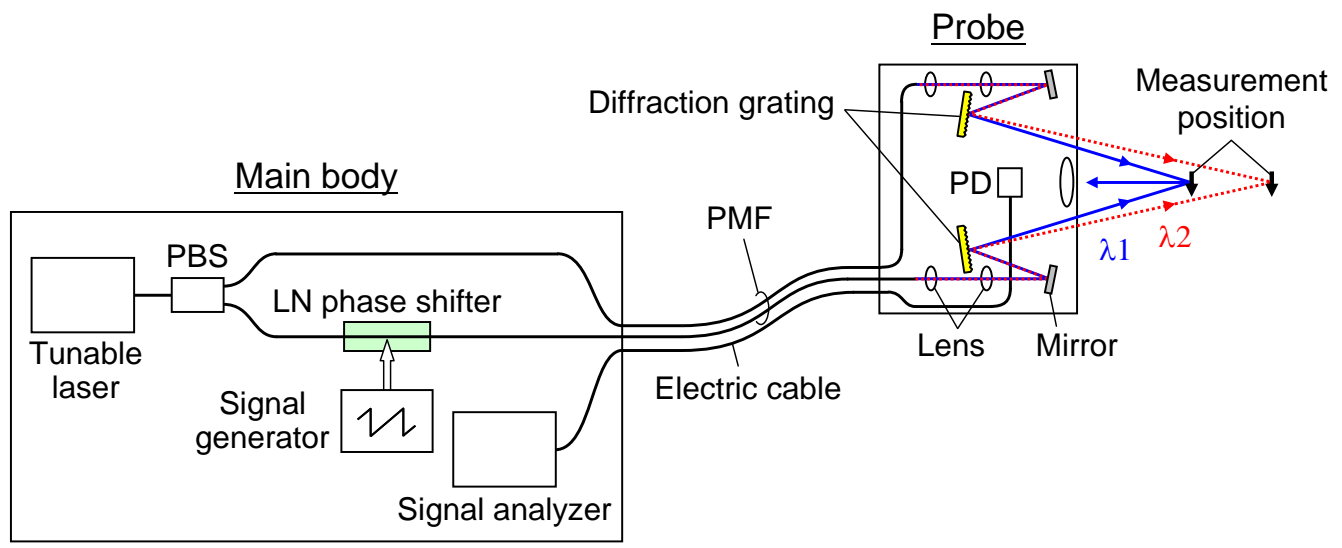

Fig. 1

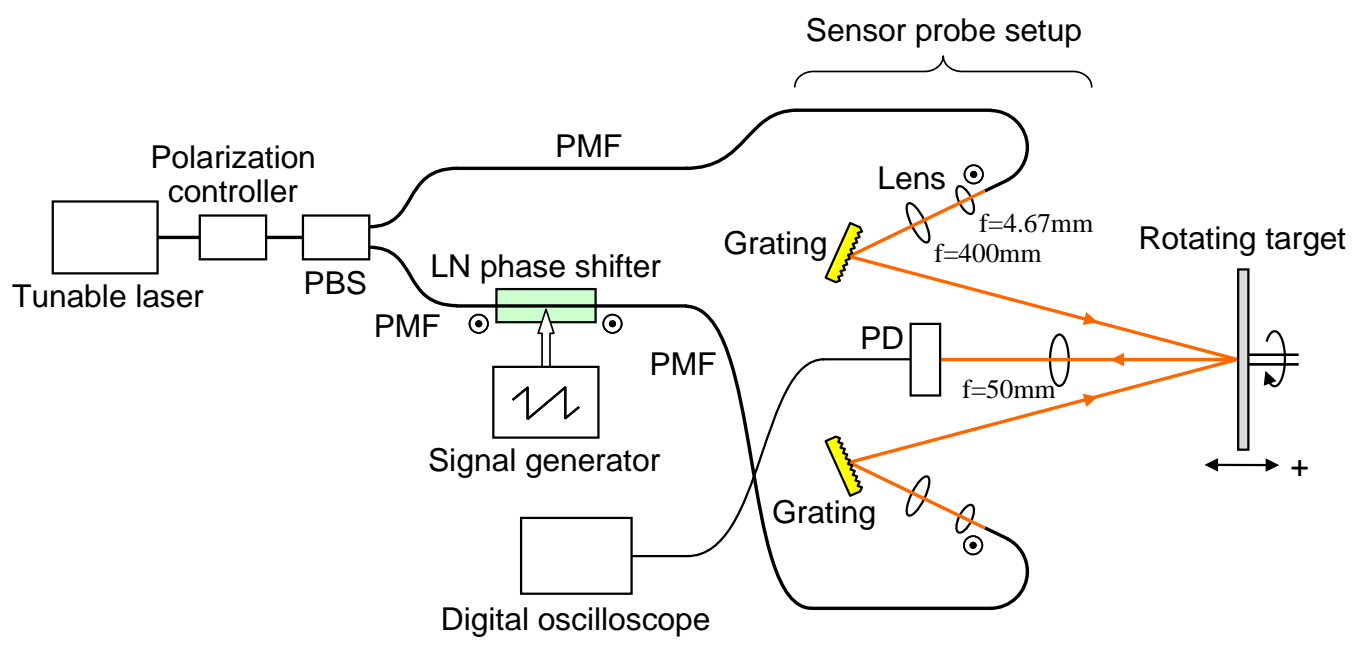

Fig. 2 


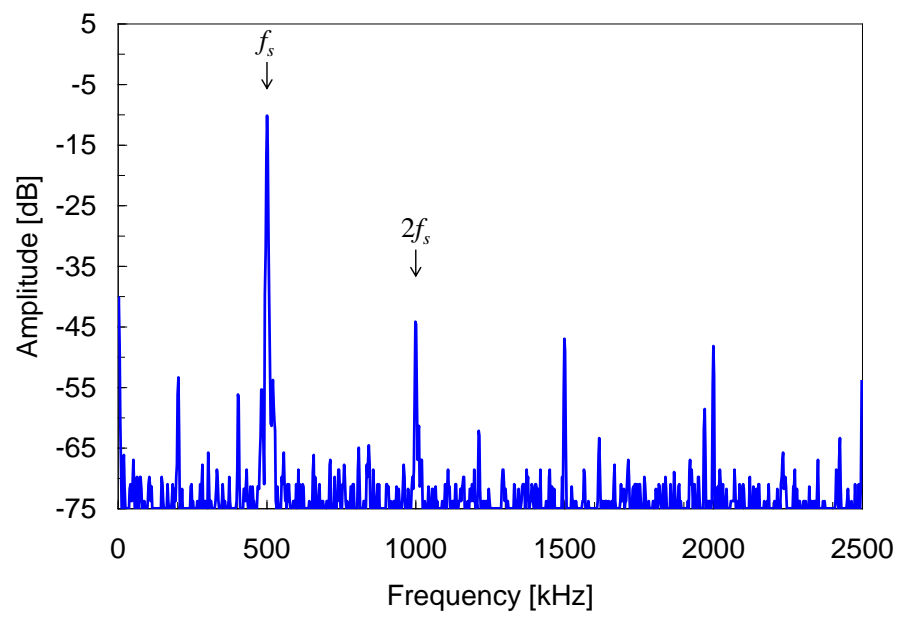

(a)

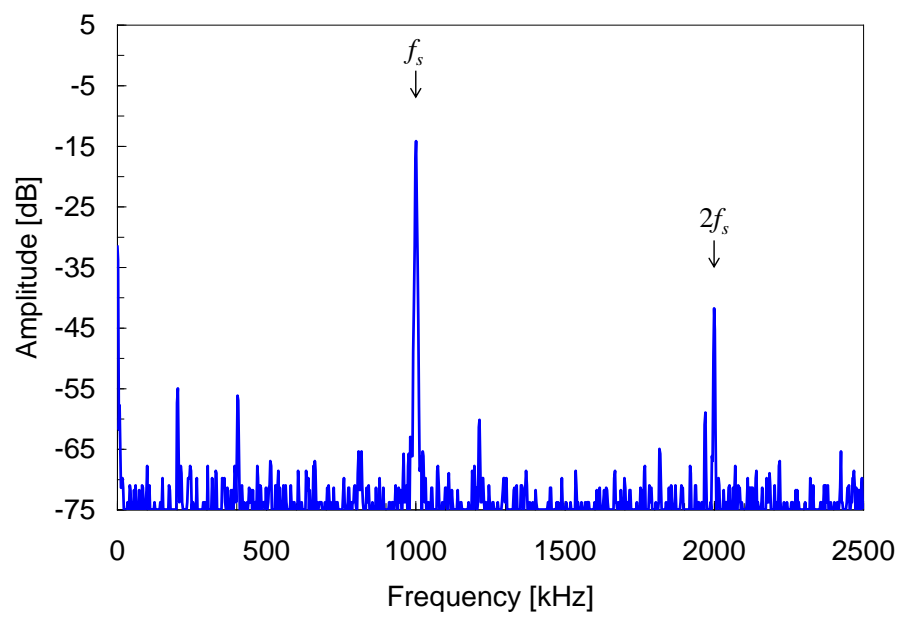

(b)

Fig. 3 


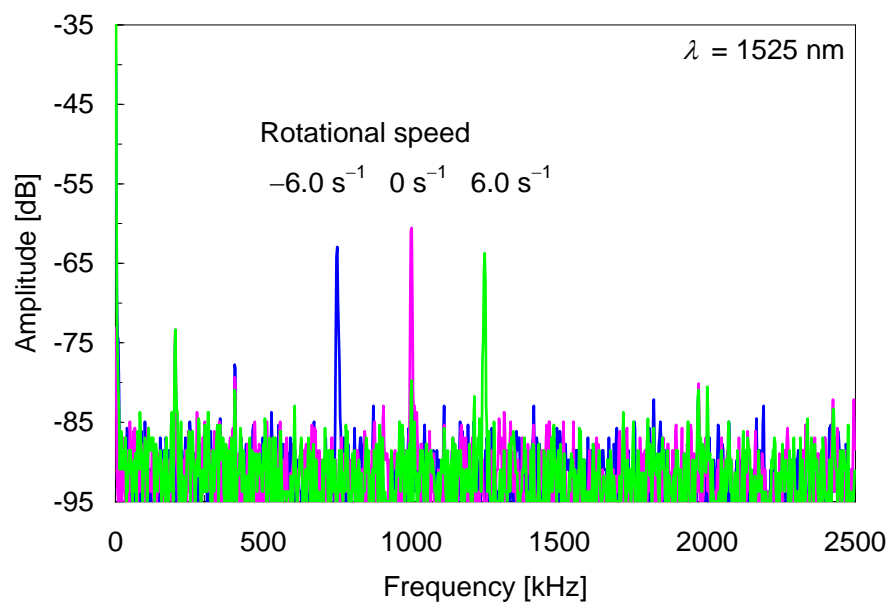

(a)

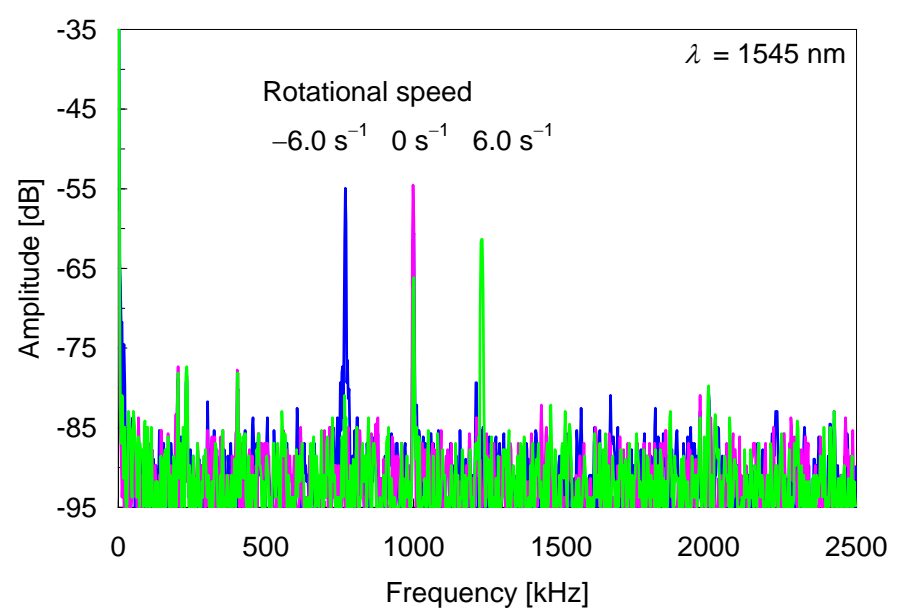

(b)

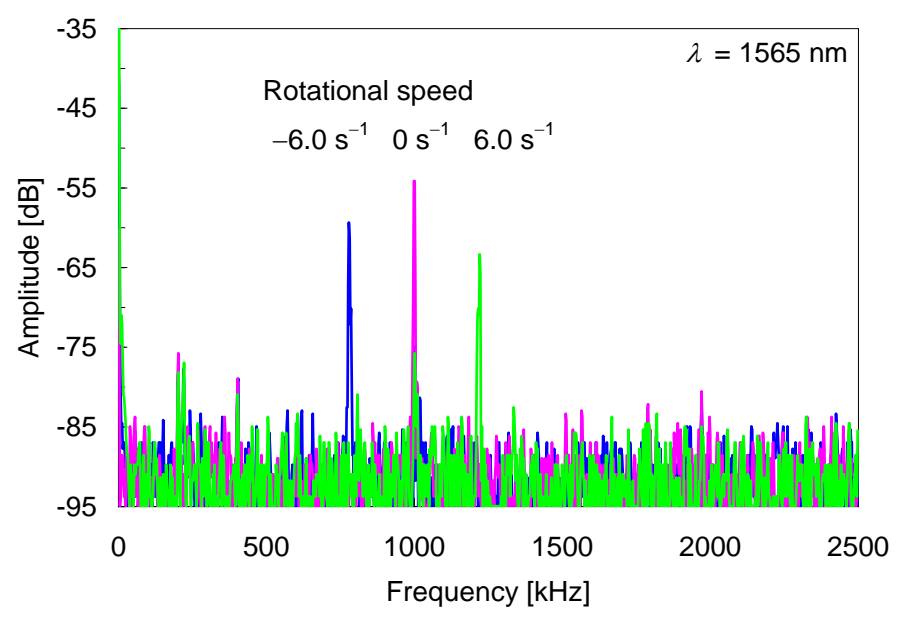

(c)

Fig. 4 


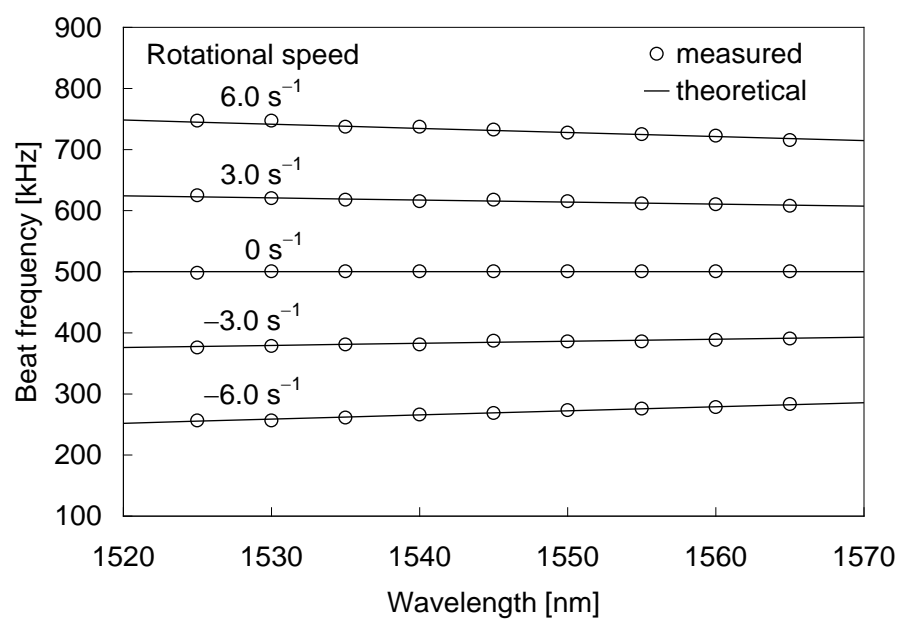

(a)

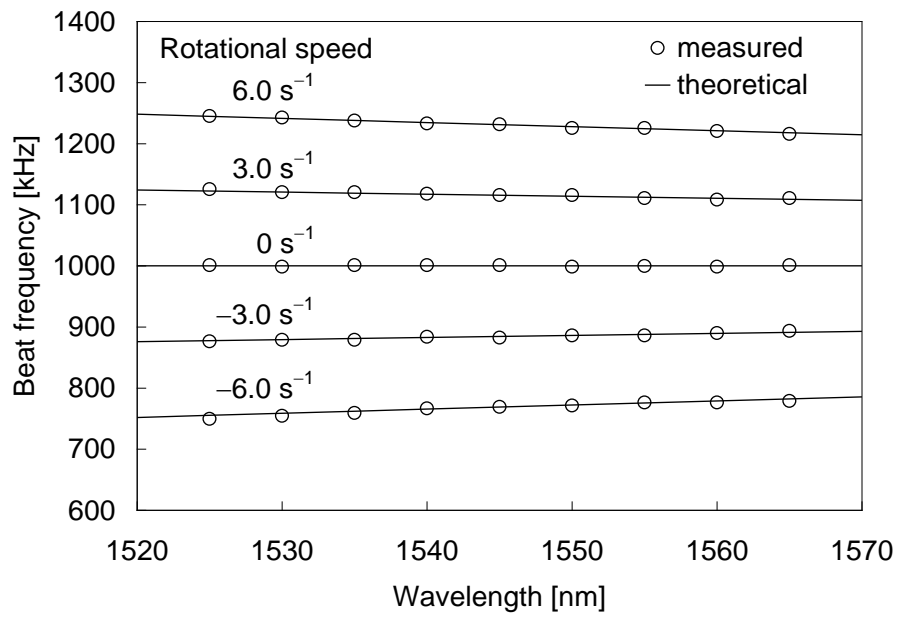

(b)

Fig. 5 


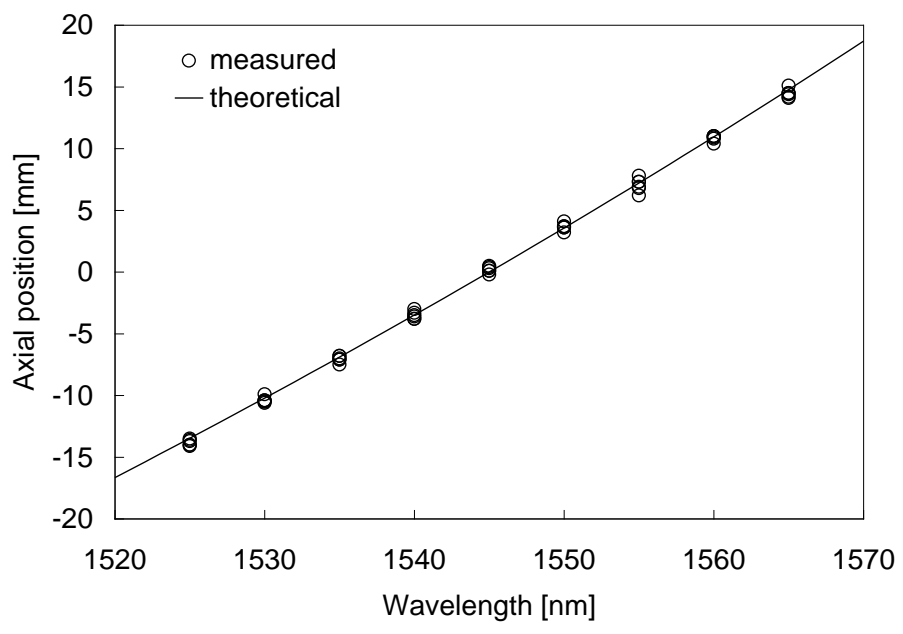

Fig. 6 